\title{
Drug-Eluting Beads Bronchial Arterial Chemoembolization in Treating Relapsed/ Refractory Small Cell Lung Cancer Patients: Results from a Pilot Study
}

\author{
Hu Lin ${ }^{1} *$ \\ Qin Wang ${ }^{1,2, *}$ \\ Fangfang Tian' \\ Rui Zhang' \\ Mi Mu' \\ Weiguo Zhao' \\ Pengtao Bao ${ }^{1,2}$ \\ 'Department of Pulmonary and Critical \\ Care Medicine, The Eighth Medical \\ Center of Chinese PLA General Hospital, \\ Pulmonary and Critical Care Medicine \\ College, Chinese PLA General Hospital, \\ Beijing, 100093, People's Republic of \\ China; ${ }^{2}$ Graduate School, Department of \\ Clinical Medicine, Hebei North \\ University, Zhangjiakou, 075000, People's \\ Republic of China
}

*These authors contributed equally to this work
Background: We aimed to explore the efficacy and tolerance of drug-eluting beads bronchial arterial chemoembolization (DEB-BACE) treatment in relapsed/refractory small cell lung cancer (SCLC) patients.

Methods: Eleven relapsed/refractory SCLC patients were enrolled and treated with DEBBACE. Then, treatment response and tumor marker levels were assessed at the first, second and sixth month post treatment. Quality of life was assessed by the EORTC QLQ-C30 scale. Progression-free survival (PFS) and overall survival (OS) were also evaluated.

Results: At the first, second and sixth month post treatment, the objective response rates were $63.6 \%, 54.5 \%$, and $36.4 \%$, respectively; and the disease control rates were $90.9 \%$, $90.9 \%$ and $54.5 \%$, respectively. In addition, the neuron-specific enolase (NSE) and progastrin-releasing peptide levels were reduced at the second and sixth month. Quality of life assessed by EORTC QLQ-C30 scale, which included subscales of general health status, functional domains, symptom domains, and single domains except for financial difficulty, was markedly improved at second month post treatment. Median values of PFS and OS were 5.1 (95\% CI: 4.1-5.9) months and 9.0 (95\% CI: 6.0-12.0) months, respectively. The ECOG score and preoperative NSE level were independent predictive factors for PFS, and age as well as lesion location were independent predictive factors for OS. Adverse events were all mild and manageable with chest pain and chest stuffiness the most common ones.

Conclusion: DEB-BACE could be a therapeutic option for relapsed/refractory SCLC patients regarding its favorable treatment response, quality of life, survival benefit and safety profile.

Keywords: small cell lung cancer, drug-eluting beads bronchial arterial chemoembolization, treatment response, survival, adverse events

\section{Introduction}

Small cell lung cancer (SCLC) is a rare but very lethal lung cancer type, and it is reported that the 5-year survival rate of SCLC patients is no more than $7 \%$. $^{1,2}$ The majority of SCLC cases could be divided into two types: limited stage and extensive stage disease. Among these two types, the former is usually treated with chemotherapy, radiation, and surgery (suitable for a very small group of patients); as for the latter, only combined chemotherapy is the mainstay of treatment. $^{3,4}$ However, regarding the most crucial treatment for SCLC, there is 
a considerable portion of patients developing resistance to chemotherapy in the clinical setting. SCLC is sensitive to the first-line chemotherapy, cisplatin and etoposide; however, it often develops chemoresistance very quickly., ${ }^{5,6}$ Moreover, many novel drugs are revealed to be inefficient in SCLC patients, such as the targeted drugs including sorafenib, gefitinib and so on. ${ }^{7,8}$ Accordingly, novel therapy is urgently required for SCLC patients.

Drug-eluting bead transarterial chemoembolization (DEB-TACE), a relatively novel chemoembolization therapy, is broadly applied in several solid tumors, mostly in liver cancers. ${ }^{9,10}$ DEB-TACE presents with many advantages in the clinical setting, such as a better response rate and improvement in reducing systemic toxicity induced by chemotherapeutics, when compared to the traditional TACE in treating liver cancer patients. ${ }^{11,12}$ Interestingly, besides liver cancers, DEB-TACE is also reported to be effective and tolerable in other solid tumors. As an example, it is reported that DEB-TACE shows comparable efficacy, more favorable tolerance, increased resection rate and less financial cost as downstaging therapy in cervical cancer patients compared to synchronous radiochemotherapy. ${ }^{13}$ When applied in lung cancers or lung metastasis, DEB-TACE is conducted via the bronchial artery, which is the drug-eluting beads bronchial arterial chemoembolization (DEB-BACE). More importantly, DEB-BACE presents with acceptable efficacy and tolerance in non-small cell lung cancer (NSCLC) patients. ${ }^{9,14}$ However, no finding has been reported regarding the efficacy and safety of DEB-BACE in a cohort of SCLC patients.

Hence, in this study, we aimed to explore the efficacy and tolerance in relapsed/refractory SCLC patients treated with drug-eluting beads bronchial arterial chemoembolization (DEB-BACE).

\section{Materials and Methods}

\section{Patients}

A total of 11 relapsed/refractory SCLC patients treated in our hospital were enrolled in this pilot study. The inclusion criteria were as follows: 1) histopathological diagnosis of SCLC; 2) age between 18 and 75 years; 3) limited-stage but not suitable for surgery; 4) relapsed or drug-resistance after first-line treatment by chemo-radiotherapy; 5) Eastern Cooperative Oncology Group score $\leq 2 ; 6$ ) life expectancy more than 6 months; 7) willing to sign informed consents. In addition, patients with any of the following conditions were excluded: 1) complicated with extensive or uncontrolled extrapulmonary metastasis; 2) white blood cell $<3 \times 10^{9} / \mathrm{L}$, platelet count $<50 \times 10^{9} / \mathrm{L}$, hemoglobin $<90 \mathrm{~g} /$ $\mathrm{L}$; 3) hepatic and renal insufficiency (creatinine $>2 \mathrm{mg} / \mathrm{L}$, aspartate transaminase (AST) and/or aminotransferase $($ AST) $>$ twice times of the normal upper limit); 4) coagulation dysfunction (International Normalized Ratio (INR) $>1.5$ ) or known hemorrhagic disease, or undergoing anticoagulant therapy; 5) active infection requiring antibiotic treatment; 6) allergy to contrast agent; 7) history of malignancies; 8) women with pregnancy or lactation. In addition, no patients with a relapsed interval more than 6 months existed in the present study. This study was approved by the Ethics Committee of The Eighth Medical Center of Chinese PLA General Hospital, Pulmonary and Critical Care Medicine College, Chinese PLA General Hospital, with an Ethical Number of 309202002280910. All enrolled patients signed the informed consents. In addition, this study was conducted in accordance with the Declaration of Helsinki.

\section{DEB-BACE Treatment}

Before operation, a bottle of Callispheres ${ }^{\circledR}$ microspheres (300-500 $\mu \mathrm{m}$, Jiangsu Hengrui Medicine Co. Ltd., Jiangsu, China) were used for loading chemotherapeutic drugs (epirubicin $80 \mathrm{mg}$ ) for 30 minutes, then non-ionic contrast agent was added in a 1:1 ratio and standing for 5 minutes. Drug-eluting beads bronchial arterial chemoembolization (DEB-BACE) treatment was carried out after preparation of microspheres. Feeding artery of tumor was identified by bronchial arteriography, then microcatheter was inserted into the branch of the feeding artery using superselective catheterization. Following that, infusion chemotherapy with etoposide $200 \mathrm{mg} / \mathrm{m}^{2}$ and cisplatin $50 \mathrm{mg} / \mathrm{m}^{2}$ was carried out. Subsequently, the prepared Callispheres ${ }^{\circledR}$ microspheres were infused into the feeding artery of tumor through a microcatheter at a speed of $1 \mathrm{~mL} / \mathrm{min}$. The chemoembolization was suspended when the blood flow in the feeding artery of tumor was slow and nearly stopped. Five minutes later, angiography was performed again to determine whether embolization was complete. If not, additional embolization with blank microspheres (without drug loading) was performed until achievement of the complete embolization (the blood flow in the tumor supply artery was stopped). 


\section{Sequential Treatment and Collaborative Treatment}

After first DEB-BACE, treatment responses were evaluated for patients. If patients achieved complete response (CR) or favorable partial response (PR), they continued to receive systemic chemotherapy regimen with etoposide $100 \mathrm{mg} /\left(\mathrm{m}^{2} \cdot \mathrm{d}\right)$ on day $1-3$ plus cisplatin $80 \mathrm{mg} /\left(\mathrm{m}^{2} \cdot \mathrm{d}\right)$ on day 1 , until they withdrew from the study. However, if patients had no response to the first DEB-BACE, or achieved an unsatisfactory PR, they underwent next DEB-BACE with a time interval of 1 month. The total times of DEB-BACE were no more than 3. Collaborative treatment included symptomatic and supportive treatment such as nutrition, analgesia, antiemetic, antipyretic, and correction of bone marrow suppression, but the following collaborative treatments were not allowed during the study: 1) other systemic antitumor therapy: intravenous chemotherapy, targeted therapy, immunotherapy, etc; 2) locally antitumor therapy: surgery, radiotherapy (including seed implantation), ablation therapy, etc.; 3) traditional Chinese medicine treatment with antitumor effect.

\section{Outcome Evaluation}

After first DEB-BACE, treatment responses were evaluated at the first, second and sixth month by computed tomography (CT) or magnetic resonance imaging (MRI) according to the revised Response Evaluation Criteria in Solid Tumors (RECIST 1.1). ${ }^{15}$ The responses were classified as CR, PR, stable disease (SD) and progressive disease (PD). Objective response rate (ORR) was calculated as the percentage of $\mathrm{CR}$ and $\mathrm{PR}$, and disease control rate (DCR) was calculated as the percentage of $\mathrm{CR}, \mathrm{PR}$ and SD. The tumor markers including neuron-specific enolase (NSE) and progastrin-releasing peptide (ProGRP) were detected before operation and at the first, second and sixth month, respectively. Quality of life was evaluated before operation and at the second month using the European Organization for Research and Treatment of Cancer (EORTC) Quality of Life Questionnaire-Core 30 (QLQ-C30). ${ }^{16}$ In addition, adverse events occurred were recorded during the study.

\section{Follow-Up and Survival Assessment}

All patients were followed up until December 2019, with a follow-up interval of 1 month. Surveillance of disease progression was conducted at each follow-up. Progressionfree survival (PFS) and overall survival (OS) were evaluated according to the follow-up records. PFS was defined as the duration from the initiation of DEB-BACE to the disease progression or death; OS was defined as the duration from the initiation of DEB-BACE to the death.

\section{Statistical Analysis}

Data were presented as number with percentage, mean with standard deviation (SD), or median with interquartile range (IQR), as appropriate. Comparison of tumor markers at multiple time points was performed by Friedman test followed by Dunn's multiple comparisons test. Comparison of QLQ-C30 before and after operation was performed by paired $t$ test. Survival analysis was carried out using Kaplan-Meier curve and Log rank test. In addition, multivariate Cox's regression analysis was performed for assessing the factors related to PFS and OS, where the independent factors were screened out by conditional forward stepwise regression method. GraphPad Prism 7.00 (GraphPad Software Inc., San Diego, California, USA) and SPSS 24.0 (IBM, Chicago, Illinois, USA) were used for diagram making and data analysis. $P$ value less than 0.05 was considered as statistical significance.

\section{Results}

\section{Clinical Features of SCLC Patients}

The $11 \mathrm{relapsed} / \mathrm{refractory}$ SCLC patients presented with a mean age of $62.8 \pm 7.2$ years with a range of 49 to 71 years (Table 1). The numbers of male and female patients were $9(81.8 \%)$ and $2(18.2 \%)$, respectively. The number of patients who had a history of smoking was $9(81.8 \%)$. In addition, the numbers of patients with ECOG score of 1 and 2 were $7(63.6 \%)$ and $4(36.4 \%)$, respectively. Numbers of patients with lesion located at the left lung and patients with lesion located at the right lung were 6 $(54.5 \%)$ and $5(45.5 \%)$, respectively. The preoperative median NSE level was 80.3 (35.7-172.6) $\mathrm{ng} / \mathrm{mL}$ with a range of $17.2 \mathrm{ng} / \mathrm{mL}-196.8 \mathrm{ng} / \mathrm{mL}$. And the preoperative median level of ProGRP was 445.0 (186.2-910.6) ng/mL, and the range was $86.7 \mathrm{ng} / \mathrm{mL}-1123.7 \mathrm{ng} / \mathrm{mL}$. Moreover, the first-line therapy was etoposide $100 \mathrm{mg} /\left(\mathrm{m}^{2} \cdot \mathrm{d}\right)$ plus cisplatin $80 \mathrm{mg} /\left(\mathrm{m}^{2} \cdot \mathrm{d}\right)$ for every patient. Besides, the detailed clinical features of each SCLC patient were presented in Table 2.

Treatment Responses and Tumor Markers At the first month post treatment, the rates of CR, PR, SD, $\mathrm{PD} /$ Death, ORR and DCR were $0.0 \%, 63.6 \%, 27.3 \%$, 
Table I Clinical Features of SCLC Patients

\begin{tabular}{|l|c|}
\hline Clinical Features & SCLC Patients (N=I I) \\
\hline Age (years) & $62.8 \pm 7.2$ \\
Mean \pm SD & $49-7 \mid$ \\
Range & \\
\hline Gender, No. (\%) & $9(81.8)$ \\
Male & $2(18.2)$ \\
Female & \\
\hline History of smoking, No. (\%) & $2(18.2)$ \\
No & $9(81.8)$ \\
Yes & \\
\hline ECOG score, No. (\%) & $7(63.6)$ \\
I & $4(36.4)$ \\
2 & \\
\hline Lesion location, No. (\%) & $6(54.5)$ \\
Left & $5(45.5)$ \\
Right & $80.3(35.7-172.6)$ \\
\hline Preoperative NSE level (ng/mL) & $17.2-196.8$ \\
Median (IQR) & \\
Range & $445.0(186.2-910.6)$ \\
\hline Preoperative ProGRP level (pg/mL) & \\
Median (IQR) & 123.7 \\
Range & \\
\hline
\end{tabular}

Abbreviations: SCLC, small cell lung cancer; SD, standard deviation; ECOG, Eastern Cooperative Oncology Group; NSE, neuron-specific enolase; IQR, interquartile range; ProGRP, progastrin-releasing peptide.

$9.1 \% / 0.0 \%, 63.6 \%$ and $90.9 \%$ in relapsed/refractory SCLC patients treated with DEB-BACE (Table 3). At the second month post treatment, rates of $\mathrm{CR}, \mathrm{PR}, \mathrm{SD}, \mathrm{PD} / \mathrm{Death}$, ORR and DCR were $18.2 \%, 36.4 \%, 36.4 \%, 0.0 \% / 9.1 \%$, $54.5 \%$ and $90.9 \%$, respectively. Moreover, at the 6 th month post treatment, the proportions of patients achieving CR, PR, SD, PD/Death, ORR and DCR were 9.1\%, $27.3 \%, 18.2 \%, 36.4 \% / 9.1 \%, 36.4 \%$ and $54.5 \%$, respectively. In terms of the tumor markers, the median NSE level was reduced at the first month $(P=0.025)$, second month $(P=0.005)$ and sixth month $(P<0.001)$ post treatment compared to that at preoperation (Figure 1A). As to another tumor marker, ProGRP level was decreased at the second month $(P=0.009)$ and sixth month $(P=$ $0.001)$, but not at the first month $(P=0.296)$ compared to that at preoperation (Figure 1B).

\section{Quality of Life}

In regard to quality of life, the data revealed that score of general health status at the second month after treatment was markedly higher compared to that before treatment in relapsed/refractory SCLC patients treated with DEBBACE $(P<0.001)$ (Table 4$)$. In addition, the functional domains including the scores of social function, emotional function, cognitive function, role function and physical function at the second months after treatment were all notably elevated compared to those before treatment (all $P<0.001)$. As for symptom domain scores, the nausea and vomiting score, pain score and fatigue score post treatment were all lower compared to that before treatment (all $P<$ 0.001). In terms of the single domains, the scores of diarrhea, constipation, loss of appetite, insomnia and shortness of breath post treatment were reduced compared to that before treatment (all $P<0.001$ ), while, the score of financial difficulty was of no difference $(P=0.844)$.

\section{Survival Profile}

Furthermore, the Kaplan-Meier curves showed that the median PFS (Figure 2A) and OS (Figure 2B) were 5.1 (95\% CI: 4.1-5.9) months and 9.0 (95\% CI: 6.0-12.0) months, respectively. In addition, the PFS $(P=0.054)$ (Figure 2C) was similar, but the OS $(P=0.032)$ (Figure 2D) was less prolonged in patients with age $\geq 65$ years compared to patients with age $<65$ years. Then, the subsequent multivariate Cox's regression analysis of factors related to PFS and OS revealed that, regarding PFS, the ECOG score 2 (vs 1) $(P=0.010)$ and preoperative NSE $\geq 5$ ULN (vs $<5 \mathrm{ULN})(P=0.033)$ were independently correlated with worse PFS in relapsed/refractory SCLC patients (Table 5). In terms of OS, the age $\geq 65$ years (vs $<65$ years $)(P=0.013)$ and lesion location at the left lung (vs right) $(P=0.046)$ independently associated with less prolonged OS.

\section{Safety Profile}

The most common adverse events in relapsed/refractory SCLC patients were fever, chest pain, chest stuffiness, bone marrow suppression, gastrointestinal effect, hemoptysis and rash. These adverse events all appeared at or at the day after DEB-BACE treatment, and were mostly mild and manageable. Among these adverse events, chest pain and chest stuffiness were the most common, followed by fever with the highest temperature of about $38^{\circ} \mathrm{C}-39^{\circ} \mathrm{C}$ and the majority of the fever patients could be treated by non-steroidal anti-inflammatory drugs or recovered by themselves. In addition, no spinal cord injury, pulmonary thrombosis, cerebrovascular event or lower limb vein thrombosis existed during or post the treatment. 
Table 2 Detailed Data of Each SCLC Patient

\begin{tabular}{|c|c|c|c|c|c|c|c|c|c|c|c|c|c|}
\hline \multirow[t]{2}{*}{ No. } & \multirow[t]{2}{*}{$\begin{array}{c}\text { Age } \\
\text { (Years) }\end{array}$} & \multirow[t]{2}{*}{ Gender } & \multirow[t]{2}{*}{$\begin{array}{l}\text { History of } \\
\text { Smoking }\end{array}$} & \multirow[t]{2}{*}{$\begin{array}{l}\text { ECOG } \\
\text { Score }\end{array}$} & \multirow[t]{2}{*}{$\begin{array}{c}\text { Lesion } \\
\text { Location }\end{array}$} & \multirow[t]{2}{*}{ TNM Stage } & \multicolumn{3}{|c|}{$\begin{array}{l}\text { Treatment } \\
\text { Response }\end{array}$} & \multirow[t]{2}{*}{$\begin{array}{c}\text { Disease } \\
\text { Progression }\end{array}$} & \multirow[t]{2}{*}{$\begin{array}{c}\text { PFS } \\
\text { (Months) }\end{array}$} & \multirow[t]{2}{*}{$\begin{array}{l}\text { Survival } \\
\text { Status }\end{array}$} & \multirow[t]{2}{*}{$\begin{array}{c}\text { OS } \\
\text { (Months) }\end{array}$} \\
\hline & & & & & & & MI & M2 & M6 & & & & \\
\hline I & 70 & Male & Yes & 2 & Left & T4N3MIc, IVB & $\mathrm{PD}$ & Dead & Dead & Yes & 1.0 & Dead & 2.0 \\
\hline 2 & 52 & Female & No & I & Right & T4N2Mla, IVA & PR & $C R$ & PR & Yes & 8.0 & Survival & 13.0 \\
\hline 3 & 61 & Male & Yes & 2 & Left & T3N2MIa, IVA & SD & SD & SD & Yes & 4.5 & Dead & 11.0 \\
\hline 4 & 66 & Male & Yes & 1 & Right & T4N3MIa, IVA & $\mathrm{PR}$ & $P R$ & PD & Yes & 5.0 & Dead & 10.0 \\
\hline 5 & 62 & Male & Yes & 1 & Left & T4N3MIc, IVB & $\mathrm{PR}$ & $P R$ & SD & Yes & 5.2 & Survival & 9.0 \\
\hline 6 & 67 & Female & No & 2 & Left & T2bN2M0, IIIA & PR & SD & PD & Yes & 3.0 & Dead & 7.0 \\
\hline 7 & 71 & Male & Yes & 2 & Right & T3N3MIc, IVB & SD & SD & PD & Yes & 3.4 & Dead & 6.7 \\
\hline 8 & 62 & Male & Yes & I & Left & T3N3M0, IIIB & PR & $P R$ & PR & Yes & 6.9 & Dead & 9.0 \\
\hline 9 & 61 & Male & Yes & 1 & Right & T3N2M0, IIIB & $P R$ & $C R$ & $C R$ & Yes & 9.2 & Survival & 11.4 \\
\hline 10 & 70 & Male & Yes & 1 & Right & T4N2MIa, IVA & $\mathrm{PR}$ & $P R$ & $P R$ & Yes & 6.3 & Dead & 8.8 \\
\hline 11 & 49 & Male & Yes & 1 & Left & T4N2MIc, IVB & $S D$ & SD & PD & Yes & 4.4 & Dead & 6.5 \\
\hline
\end{tabular}

Abbreviations: SCLC, small cell lung cancer; ECOG, Eastern Cooperative Oncology Group; MI, I month after operation; M2, 2 months after operation; M6, 6 months after operation; CR, complete response; PR, partial response; SD, stable disease; PD, progressive disease; PFS, progression-free survival; OS, overall survival.

Table 3 Treatment Response

\begin{tabular}{|l|c|c|c|c|c|c|}
\hline \multirow{2}{*}{ Time Interval } & \multicolumn{4}{|c|}{ Treatment Response, No. (\%) } & \multirow{2}{*}{ ORR } & \multirow{2}{*}{ DCR } \\
\cline { 2 - 6 } & CR & PR & SD & PD/Death & & \\
\hline Ist month & $0(0.0)$ & $7(63.6)$ & $3(27.3)$ & $1(9.1) / 0(0.0)$ & $7(63.6)$ & $10(90.9)$ \\
2nd month & $2(18.2)$ & $4(36.4)$ & $4(36.4)$ & $0(0.0) / 1(9.1)$ & $6(54.5)$ & $10(90.9)$ \\
6th month & I (9.1) & $3(27.3)$ & $2(18.2)$ & $4(36.4) / I(9.1)$ & $4(36.4)$ & $6(54.5)$ \\
\hline
\end{tabular}

Abbreviations: $C R$, complete response; PR, partial response; SD, stable disease; PD, progressive disease; ORR, objective response rate; DCR, disease control rate.

Additionally, some serious adverse events, such as the spinal cord injury, did not occur during and after the treatment.

\section{Presentation of a Successful Case}

The image examinations of one patient were displayed in Figure 3. During angiography in the left bronchial artery, the tumor was selected under X-ray via identifying the dyed area (Figure 3A), which disclosed that the bronchial branches from the internal thoracic artery were responsible for blood supply of the targeted tumor in the left lung (Figure 3B). Therefore, the superselective catheterization was performed on the bronchial branches from the internal thoracic artery (Figure 3C). Previously, the patient was treated with chemotherapy, and CT image showed that the tumor at the upper lobe of left lung was reduced and maintained stable after chemotherapy (Figure 3D). However, the reexamination of this patient revealed a progression of the tumor (Figure 3E); hence, the patient subsequently received DEB-BACE treatment. At the first month post the DEB-BACE treatment, reexamination of the patient showed reduction of the tumor in the upper lobe of left lung and a decrease of left pleural effusion (Figure 3F).

\section{Discussion}

The dismal prognosis of SCLC patients is largely ascribed to that it is sensitive to chemotherapy but quickly develops chemoresistance, which has remained to be one of the most essential problems in SCLC management. Many mechanisms have been proposed regarding the development of chemoresistance in SCLC patients, for instance, the overexpressions of multidrug resistance (MDR)-related factors, dysregulation of intracellular enzyme system, abnormal apoptotic functions of the tumor cells and so on; however, most of these still need validation by experiments. ${ }^{17-22}$ Meanwhile, many other non-first line therapies are introduced in the management of SCLC patients in practice. However, according to previous studies, efficiency, and safety of DEB-BACE in treating the SCLC patients have not been reported. Based on the fact that DEB-BACE is efficient and tolerable in treating lung 
A

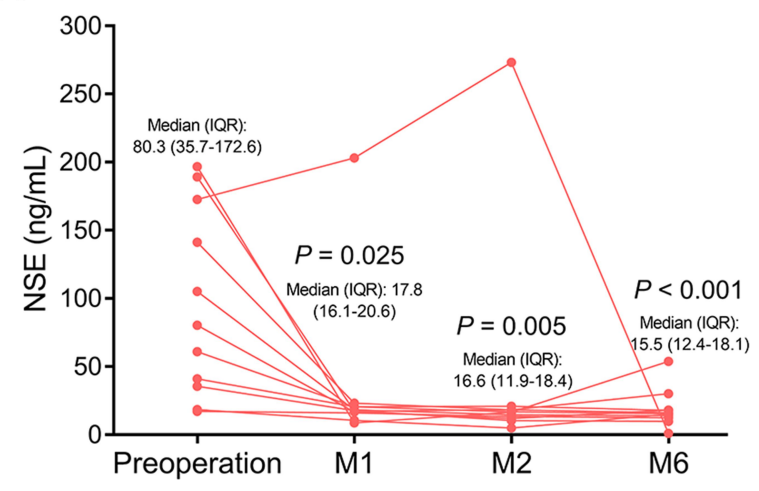

B

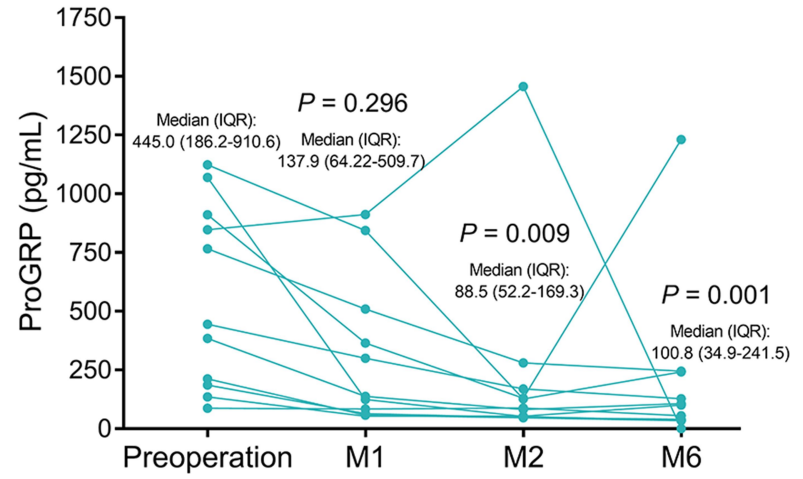

Figure I Decrease of tumor marker levels post DEB-BACE treatment. The NSE level (A) and ProGRP level (B) at preoperation, I st month, 2nd month and 6th month post DEB-BACE treatment in relapsed/refractory SCLC patients.

Abbreviations: DEB-BACE, drug-eluting beads bronchial arterial chemoembolization; NSE, neuron-specific enolase, ProGRP, progastrin-releasing peptide; SCLC, small cell lung cancer; IQR, interquartile range.

cancers other than SCLC, we conducted the present study to preliminarily explore the efficacy, quality of life and survival of relapsed/refractory SCLC patients treated by DEB-BACE. Subsequently, we found that in relapsed/ refractory SCLC patients treated with DEB-BACE: 1) the ORR and DCR were favorable and the tumor marker levels were decreased at first, second, and sixth month post treatment. 2) The quality of life was markedly improved at the second month post treatment. 3) Median values of PFS and OS were 5.1 (95\% CI: 4.1-5.9) months and 9.0 (95\% CI: 6.0-12.0) months, besides, ECOG score and preoperative NSE level were independent predictive factors for PFS, and age as well as lesion location were independent predictive factors for OS. 4) Adverse events post treatment were all mild and manageable with the most frequent ones being chest pain and chest stuffiness.

Table 4 QLQ-C30 Score Before and at 2 Months After Treatment

\begin{tabular}{|c|c|c|c|}
\hline \multirow[t]{2}{*}{ Items } & \multicolumn{2}{|c|}{ QLQ-C30 Score, Mean \pm SD } & \multirow[t]{2}{*}{$P$ value } \\
\hline & Before Treatment & Two Months After Treatment & \\
\hline General health status & $43.5 \pm 9.8$ & $52.5 \pm 9.1$ & $<0.001$ \\
\hline \multicolumn{4}{|l|}{ Functional domains } \\
\hline Social function & $50.1 \pm 9.2$ & $62.2 \pm 8.7$ & $<0.001$ \\
\hline Emotional function & $54.8 \pm 6.6$ & $75.5 \pm 6.0$ & $<0.001$ \\
\hline Cognitive function & $63.7 \pm 6.4$ & $75.2 \pm 6.2$ & $<0.001$ \\
\hline Role function & $63.5 \pm 6.8$ & $76.8 \pm 6.4$ & $<0.001$ \\
\hline Physical function & $59.9 \pm 9.7$ & $70.3 \pm 11.0$ & $<0.001$ \\
\hline \multicolumn{4}{|l|}{ Symptom domains } \\
\hline Nausea and vomiting & $52.2 \pm 9.0$ & $42.8 \pm 6.8$ & $<0.001$ \\
\hline Pain & $26.8 \pm 5.6$ & $14.0 \pm 2.5$ & $<0.001$ \\
\hline Fatigue & $45.5 \pm 11.7$ & $32.5 \pm 9.2$ & $<0.001$ \\
\hline \multicolumn{4}{|l|}{ Single domains } \\
\hline Diarrhea & $42.1 \pm 6.4$ & $34.9 \pm 5.1$ & $<0.001$ \\
\hline Constipation & $40.2 \pm 10.7$ & $33.4 \pm 10.1$ & $<0.001$ \\
\hline Loss of appetite & $42.6 \pm 8.7$ & $31.8 \pm 9.4$ & $<0.001$ \\
\hline Insomnia & $25.6 \pm 5.3$ & $20.4 \pm 3.7$ & $<0.001$ \\
\hline Shortness of breath & $21.6 \pm 6.4$ & $11.5 \pm 1.8$ & $<0.001$ \\
\hline Financial difficulty & $45.4 \pm 6.0$ & $44.9 \pm 7.9$ & 0.844 \\
\hline
\end{tabular}

Abbreviations: QLQ-C30, Quality of Life Questionnaire-Core 30; SD, standard deviation. 
A

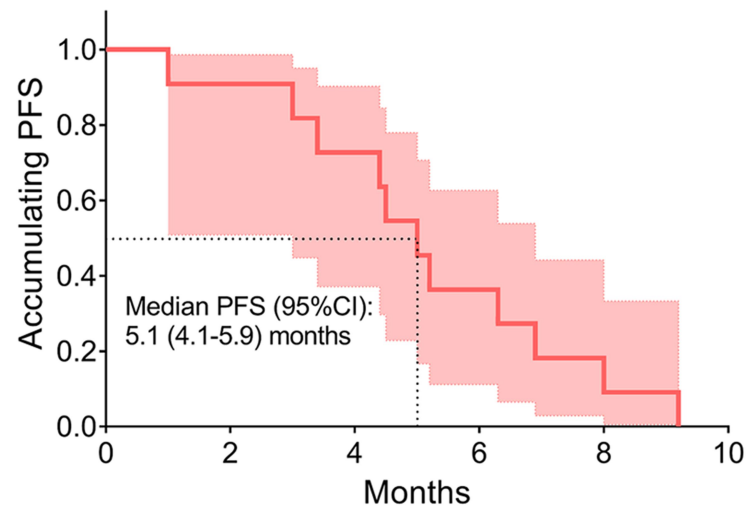

C

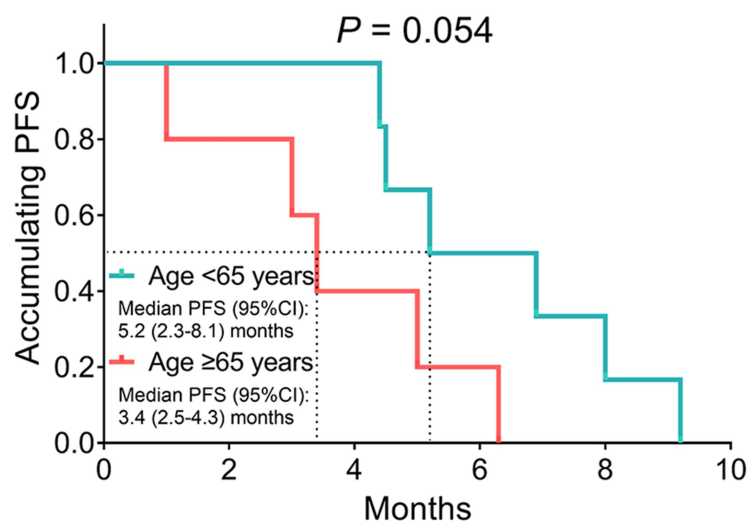

B

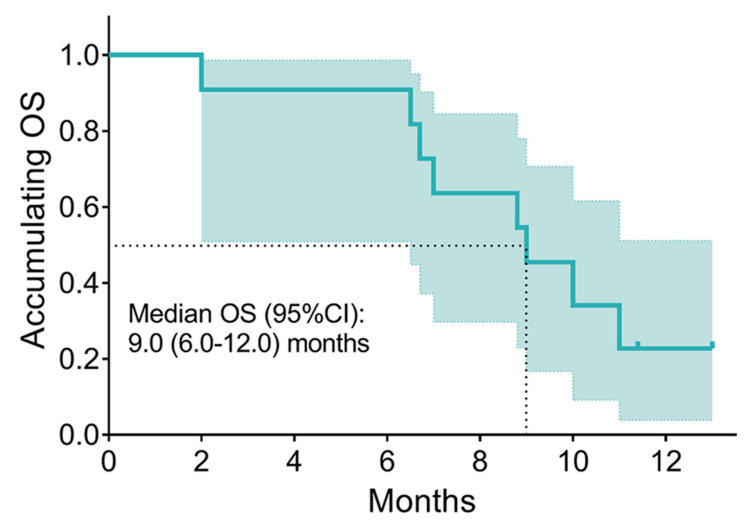

D

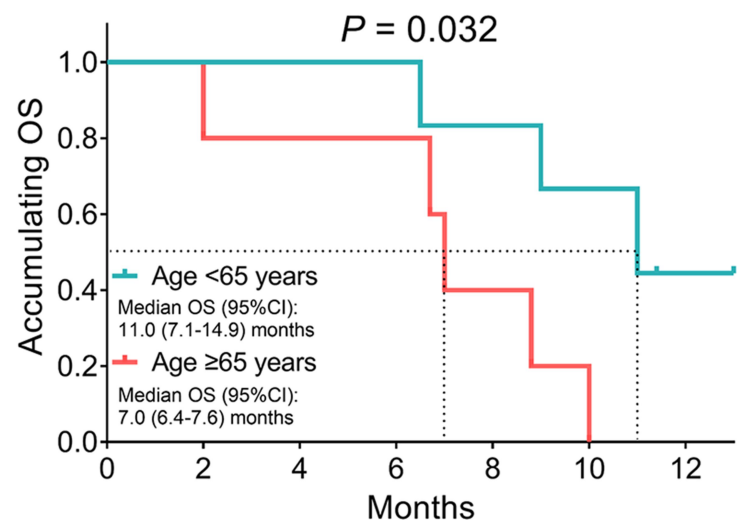

Figure 2 PFS and OS. The median values of PFS (A) and OS (B) in total patients, and the correlations of PFS (C) as well as OS (D) with age. Abbreviations: PFS, progression-free survival; OS, overall survival; $95 \% \mathrm{Cl}, 95 \%$ confidence interval.

Generally, DEB-TACE is predominantly applied in liver cancers and metastatic tumor in the liver; however, in recent years, increasing studies disclose that it could also be utilized in other solid tumors. ${ }^{9-12}$ Most importantly, in regard to its application in lung cancers or metastasis to the lung, a prior study reveals that in six NSCLC patients who are not able to receive standard treatment, the ORR and DCR are respectively $50.0 \%$ and $100.0 \%$ at 2 months, $50.0 \%$ and $83.3 \%$ at 4 months, $50.0 \%$ and $66.7 \%$ at 6 months post DEB-BACE treatment. ${ }^{23}$ Another study elucidates that in 52 patients with lung metastasis from other solid tumors, the DEB-BACE treatment achieves PR, SD and PD of $30.8 \%, 21.2 \%$ and $48.0 \%$, respectively. ${ }^{24}$ These studies indicate relatively good treatment responses by DEB-BACE in NSCLC and lung metastasis. To the best of our knowledge, the present study was the first to detect the efficacy of DEB-BACE in a cohort of relapsed/refractory SCLC patients, which disclosed that the rates of ORR $(63.6 \%, 54.5 \%$ and $36.4 \%)$ and DCR $(90.9 \%, 90.9 \%$ and $54.5 \%)$ were all favorable at the first, second and sixth month post treatment. As for possible explanations to this result, we hypothesized that, first, one of the mechanisms of DEB-BACE treatment was to embolize the tumor supplying artery and lead to the necrosis inside the tumor, which was a different pathway compared to simple chemotherapeutics infusion, therefore enhanced the treatment efficacy. ${ }^{25}$ Second, when compared to the traditional chemotherapy through intravenous infusion, the directional and continuous release of chemotherapeutics to the targeted tumor by DEB-BACE possibly enhanced the cytotoxicity inside the tumor. Hence, the DEB-BACE treatment could realize favorable treatment responses in relapsed/refractory SCLC patients in our study.

Psychological stress induced by the high mortality rate, clinical symptoms and many side effects from treatments all contribute to the decline in quality of life in lung cancer patients, which of course include the SCLC patients. In terms of the lung cancer patients treated by DEB-TACE, a previous study elucidates that the global quality of life, 
Table 5 Cox's Regression Analysis of Factors Related to PFS and OS

\begin{tabular}{|c|c|c|c|c|c|}
\hline \multirow[t]{2}{*}{ Items } & \multicolumn{5}{|c|}{ Multivariate Cox's Regression Analysis (Forward Stepwise) } \\
\hline & Beta & S.E. & Wald & $P$ value & HR $(95 \% \mathrm{Cl})$ \\
\hline \multicolumn{6}{|l|}{ PFS } \\
\hline ECOG score (2 vs I) & 3.775 & $1.46 \mathrm{I}$ & 6.676 & 0.010 & 43.609 (2.488-764.377) \\
\hline Preoperative NSE ( $\geq 5$ ULN vs $<5$ ULN) & 2.412 & 1.130 & 4.558 & 0.033 & $11.159(1.219-102.172)$ \\
\hline \multicolumn{6}{|l|}{ os } \\
\hline Age ( $\geq 65$ years vs $<65$ years) & 2.608 & 1.052 & 6.149 & 0.013 & $13.576(1.727-106.699)$ \\
\hline Lesion location (left vs right) & 1.826 & 0.917 & 3.965 & 0.046 & $6.211(1.029-37.489)$ \\
\hline
\end{tabular}

Abbreviations: PFS, progression-free survival; OS, overall survival; HR, hazard ratio; Cl, confidence interval; ECOG, Eastern Cooperative Oncology Group; NSE, neuronspecific enolase; ULN, upper limit of normal.

physical function and emotional function assessed by QLQ-C30 scale are improved at the second month post DEB-BACE treatment in NSCLC patients. ${ }^{9}$ In our study, the general health status, functional status, symptoms, and single domains of quality of life in QLQ-C30 were all greatly enhanced at the second month post DEB-BACE treatment in relapsed/refractory SCLC patients. We presumed that the probable logics underlying the improvement of quality of life by DEB-BACE treatment may include the followings. Patients' symptoms were ameliorated after treatment, such as chest pain, dysphagia and vomiting, etc, which normally would improve the patients' quality of life. Moreover, patients' physical function was also recovering along with the reduction of tumor burden, and this could also largely contribute to patients' quality of life improvement.

Previous studies have shown that DEB-BACE is beneficial regarding the improvement of lung cancer patients'
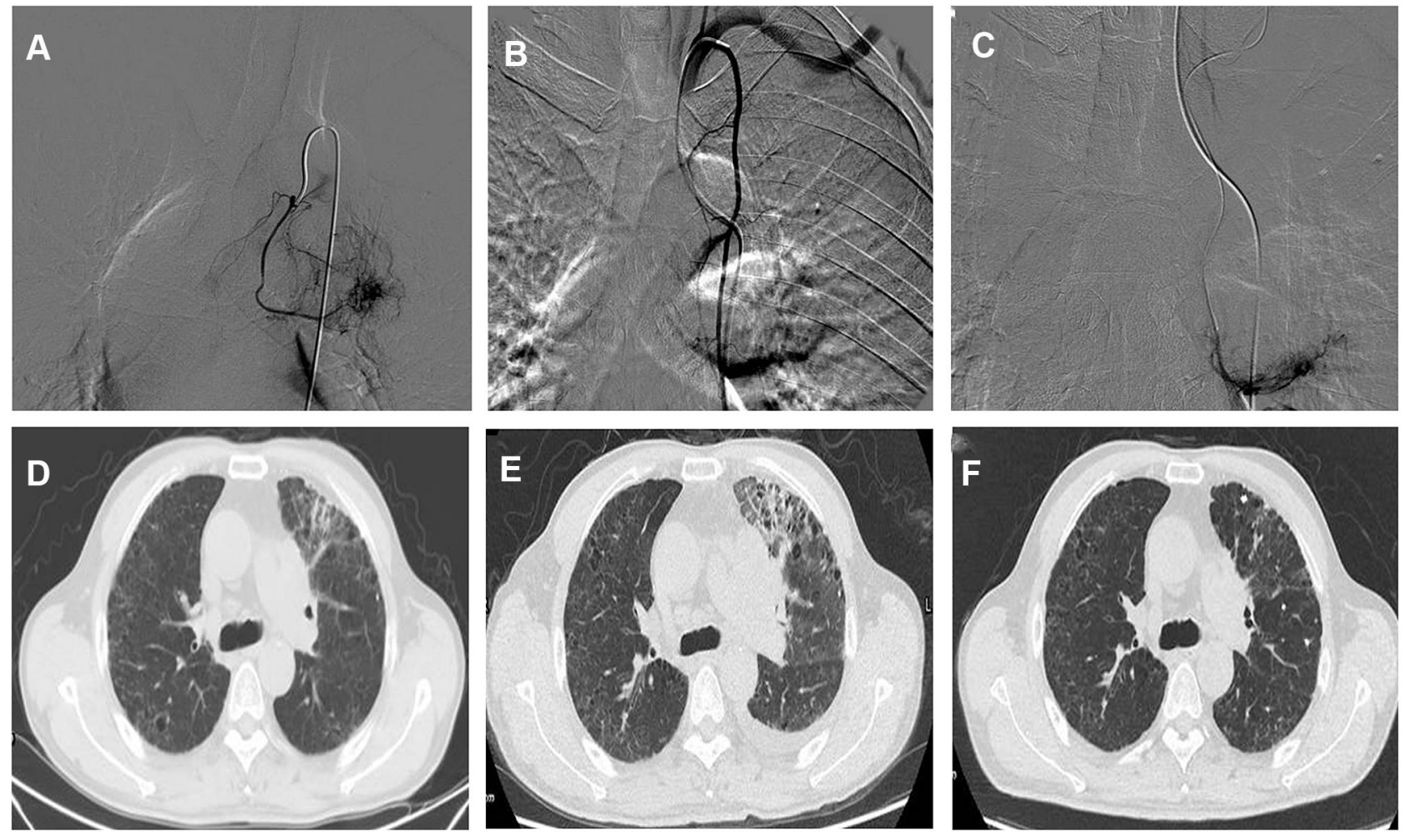

Figure 3 Angiography and chest CT images of a SCLC patient. The images of angiography in the lung during DEB-BACE (A-C), CT image of the reduction of lesion after chemotherapy in the left lung (D), CT image showing a progression of the lesion after chemotherapy (E), and the CT image showing reduction of lesion after DEB-BACE treatment (F).

Abbreviations: CT, computed tomography; DEB-BACE, drug-eluting beads bronchial arterial chemoembolization; SCLC, small cell lung cancer. 
survival. Such as, a prospective cohort study reveals that, in advanced lung cancer patients (squamous cell carcinoma, adenocarcinoma and others), DEB-BACE realizes better 3-month PFS rate (96.7\% vs 73.3\%), 6-month PFS rate $(87.5 \%$ vs $57.1 \%)$ and 6 -month OS rate $(87.5 \%$ vs $52.7 \%$ ) compared to bronchial arterial infusion chemotherapy. $^{26}$ Another cohort study illustrates that using DEB-BACE for unresectable lung metastasis from other solid tumors, the mean survival time is 17 months and the median survival period is 21.1 months. $^{24}$ In the present study, we found that DEB-BACE accomplished a median PFS of 5.1 (4.1-5.9) months and a median OS of 9.0 (6.0-12.0) months in relapsed/refractory SCLC patients, which was a favorable survival profile. Furthermore, we presumed that this result could be caused by the good treatment responses induced by DEB-BACE, which considerably enhanced the patients' survival time due to the restraining from tumor progression. In addition, we also discovered that ECOG score and preoperative NSE level were independent predictive factors for PFS, and age as well as lesion location were independent predictive factors for OS in relapsed/refractory SCLC patients treated with DEB-BACE. As to more advanced ECOG status and older age, they indicated a worse physical function, which may interfere with the efficacy of treatment due to a decreased tolerance to the chemotherapeutics, increased preoperative NSE level indicated a more advanced disease condition, and many studies have revealed that NSE level negatively correlated with SCLC patients' prognosis. ${ }^{27,28}$ As for the lesion location, right lung had a larger size and more abundant blood supply than the left lung, which probably made the effect of embolization and chemotherapeutics more satisfying when compared to the left lung, and thus enhanced the treatment efficiency. As discussed above, we presumed that the aforementioned facts may be possible explanations of the prognostic value of the four factors found in our study, which, however, needed to be validated by more studies. In addition, in regard to the clinical value of these results, it could be that the relapsed/refractory SCLC patients with higher ECOG score, preoperative NSE level, age older than 65 years and lesion location at the left lung were less suitable for receiving DEB-BACE treatment. Nonetheless, these should be established by large-scale clinical studies. Furthermore, DEB-BACE also presented with a favorable safety profile in relapsed/ refractory SCLC patients, which displayed that most of the adverse events were mild and manageable post treatment, with the most common ones including chest pain and chest stuffiness.

The present study was the first to explore the efficacy, safety and quality of life in relapsed/refractory SCLC patients treated with DEB-BACE; however, there were several limitations that should be discussed. This was a pilot study; thus, the small sample size could largely reduce the statistical power; also, it blocked our way to add more analysis in our study. In addition, this was an observational study, which could result in some bias.

In conclusion, DEB-BACE could be a therapeutic option for relapsed/refractory SCLC patients regarding its favorable treatment response, quality of life, survival benefit and safety profile.

\section{Ethics Approval and Consent to Participate}

This study was approved by the Ethics Committee of our hospital, with an Ethical Number of 309202002280910. All enrolled patients signed the informed consents.

\section{Funding and Acknowledgments}

This study was supported by Logistic Support Department of CentralMilitary Commission Health Care Project (No.21BJZ42) and National Natural Science Foundation of Beijing(No.7212104).

\section{Disclosure}

The abstract of this paper was presented at the European Conference on Interventional Oncology 2021 as a poster presentation with interim findings. The poster's abstract was published in Journal Cardiovascular and Interventional Radiology volume 44, pages 1-64 (2021): [https://link.springer.com/article/10.1007/s00270-02102819-z]. The authors declare that they have no competing interest.

\section{References}

1. Ferlay J, Shin HR, Bray F, Forman D, Mathers C, Parkin DM. Estimates of worldwide burden of cancer in 2008: GLOBOCAN 2008. Int $J$ Cancer. 2010;127(12):2893-2917. doi:10.1002/ ijc. 25516

2. Siegel RL, Miller KD, Jemal A. Cancer statistics, 2018. CA Cancer J Clin. 2018;68(1):7-30. doi:10.3322/caac.21442

3. Kalemkerian GP. Small cell lung cancer. Semin Respir Crit Care Med. 2016;37(5):783-796. doi:10.1055/s-0036-1592116

4. Kalemkerian GP, Schneider BJ. Advances in small cell lung cancer. Hematol Oncol Clin North Am. 2017;31(1):143-156. doi:10.1016/j. hoc.2016.08.005 
5. Kohara H, Tabata M, Kiura K, et al. Synergistic effects of topoisomerase I inhibitor, 7-ethyl-10-hydroxycamptothecin, and irradiation in a cisplatin-resistant human small cell lung cancer cell line. Clin Cancer Res. 2002;8(1):287-292.

6. Xiang H, Long L, Yao Y, Fang Z, Zhang Z, Zhang Y. CalliSpheres drug-eluting bead transcatheter arterial chemoembolization presents with better efficacy and equal safety compared to conventional TACE in treating patients with hepatocellular carcinoma. Technol Cancer Res Treat. 2019;18:1533033819830751.

7. Sharma N, Pennell N, Nickolich M, et al. Phase II trial of sorafenib in conjunction with chemotherapy and as maintenance therapy in extensive-stage small cell lung cancer. Invest New Drugs. 2014;32 (2):362-368. doi:10.1007/s10637-013-0061-6

8. Lu HY, Wang XJ, Mao WM. Targeted therapies in small cell lung cancer. Oncol Lett. 2013;5(1):3-11.

9. Bie Z, Li Y, Li B, Wang D, Li L, Li X. The efficacy of drug-eluting beads bronchial arterial chemoembolization loaded with gemcitabine for treatment of non-small cell lung cancer. Thorac Cancer. 2019;10 (9):1770-1778. doi:10.1111/1759-7714.13139

10. Ni JY, Sun HL, Chen YT, et al. Drug-eluting bead transarterial chemoembolization in the treatment for unresectable soft tissue sarcoma refractory to systemic chemotherapy: a preliminary evaluation of efficacy and safety. J Cancer Res Clin Oncol. 2018;144 (1):157-163. doi:10.1007/s00432-017-2530-3

11. Ma Y, Zhao C, Zhao H, et al. Comparison of treatment efficacy and safety between drug-eluting bead transarterial chemoembolization with CalliSpheres((R)) microspheres and conventional transarterial chemoembolization as first-line treatment in hepatocellular carcinoma patients. Am J Transl Res. 2019;11(12):7456-7470.

12. Wen P, Chen SD, Wang JR, Zeng YH. Comparison of treatment response and survival profiles between drug-eluting bead transarterial chemoembolization and conventional transarterial chemoembolization in Chinese hepatocellular carcinoma patients: a Prospective Cohort Study. Oncol Res. 2019;27(5):583-592. doi:10.3727/ 096504018X15368325811545

13. Song J, Chen W, Zhu X, et al. Short-term efficacy, safety, and cost-effectiveness of transarterial chemoembolization with drug-eluting beads versus synchronous radiochemotherapy for cervical cancer. Int J Gynaecol Obstet. 2019;147(1):29-35. doi:10.1002/ ijgo. 12888

14. Yu G, Hu J. Drug-eluting beads bronchial arterial chemoembolization as a neoadjuvant treatment for squamous non-small cell lung cancer. Postgrad Med. 2020;132(6):568-571. doi:10.1080/ 00325481.2020 .1761711

15. Eisenhauer EA, Therasse P, Bogaerts J, et al. New response evaluation criteria in solid tumours: revised RECIST guideline (version 1.1). Eur $J$ Cancer. 2009;45(2):228-247. doi:10.1016/j. ejca.2008.10.026

16. Aaronson NK, Ahmedzai S, Bergman B, et al. The European Organization for Research and Treatment of Cancer QLQ-C30: a quality-of-life instrument for use in international clinical trials in oncology. J Natl Cancer Inst. 1993;85(5):365-376. doi:10.1093/ jnci/85.5.365
17. Dingemans AM, Witlox MA, Stallaert RA, van der Valk P, Postmus PE, Giaccone G. Expression of DNA topoisomerase IIalpha and topoisomerase IIbeta genes predicts survival and response to chemotherapy in patients with small cell lung cancer. Clin Cancer Res. 1999;5(8):2048-2058.

18. Sohn JW, Lee SY, Lee SJ, et al. MDR1 polymorphisms predict the response to etoposide-cisplatin combination chemotherapy in small cell lung cancer. Jpn J Clin Oncol. 2006;36(3):137-141. doi:10.1093/ jjco/hyi231

19. Gemba K, Ueoka H, Kiura K, Tabata M, Harada M. Immunohistochemical detection of mutant p53 protein in small-cell lung cancer: relationship to treatment outcome. Lung Cancer. 2000;29(1):23-31. doi:10.1016/S0169-5002(00)00098-2

20. Gardner EE, Lok BH, Schneeberger VE, et al. Chemosensitive relapse in small cell lung cancer proceeds through an EZH2-SLFN11 axis. Cancer Cell. 2017;31(2):286-299. doi:10.1016/j.ccell.2017.01.006

21. Qiu Z, Zhu W, Meng H, et al. CDYL promotes the chemoresistance of small cell lung cancer by regulating $\mathrm{H} 3 \mathrm{~K} 27$ trimethylation at the CDKN1C promoter. Theranostics. 2019;9(16):4717-4729. doi: $10.7150 /$ thno.33680

22. Zeng F, Wang Q, Wang S, et al. Linc00173 promotes chemoresistance and progression of small cell lung cancer by sponging miR-218 to regulate Etk expression. Oncogene. 2020;39(2):293-307. doi:10.1038/s41388-019-0984-2

23. Wang Z, Niu H, Li Z, et al. Superselective arterial embolization with drug-loaded microspheres for the treatment of unresectable breast cancer. Gland Surg. 2019;8(6):740-747. doi:10.21037/gs.2019.12.06

24. Vogl TJ, Lehnert T, Zangos S, et al. Transpulmonary chemoembolization (TPCE) as a treatment for unresectable lung metastases. Eur Radiol. 2008;18(11):2449-2455. doi:10.1007/s00330-008-1056-0

25. Baylatry MT, Pelage JP, Wassef M, et al. Pulmonary artery chemoembolization in a sheep model: evaluation of performance and safety of irinotecan eluting beads (DEB-IRI). J Biomed Mater Res B Appl Biomater. 2011;98(2):351-359. doi:10.1002/jbm.b.31858

26. Shang B, Li J, Wang X, et al. Clinical effect of bronchial arterial infusion chemotherapy and CalliSpheres drug-eluting beads in patients with stage II-IV lung cancer: a prospective cohort study. Thorac Cancer. 2020;11(8):2155-2162. doi:10.1111/17597714.13522

27. Zhou M, Wang Z, Yao Y, Zhou H, Liu M, Sun J. Neuron-specific enolase and response to initial therapy are important prognostic factors in patients with small cell lung cancer. Clin Transl Oncol. 2017;19(7):865-873. doi:10.1007/s12094-017-1617-2

28. Huang Z, Xu D, Zhang F, Ying Y, Song L. Pro-gastrin-releasing peptide and neuron-specific enolase: useful predictors of response to chemotherapy and survival in patients with small cell lung cancer. Clin Transl Oncol. 2016;18(10):1019-1025. doi:10.1007/ s12094-015-1479-4

\section{Publish your work in this journal}

Cancer Management and Research is an international, peer-reviewed open access journal focusing on cancer research and the optimal use of preventative and integrated treatment interventions to achieve improved outcomes, enhanced survival and quality of life for the cancer patient.
The manuscript management system is completely online and includes a very quick and fair peer-review system, which is all easy to use. Visit http://www.dovepress.com/testimonials.php to read real quotes from published authors. 